\title{
Second-order achromat design based on FODO cell
}

\author{
Yipeng Sun (孙一鹏)* \\ SLAC National Accelerator Laboratory, Menlo Park, California 94025, USA
}

(Received 16 February 2011; published 13 June 2011)

\begin{abstract}
Two dipole doglegs are widely used to translate the beam axis horizontally or vertically. Quadrupoles are placed between the two consecutive dipoles to match first-order dispersion and provide betatron focusing. Similarly, a four dipole chicane is usually employed to form a bypass region, where the beam axis is transversely shifted first, then translated back to the original axis. In order to generate an isochronous section, quadrupoles are again needed to tune the first-order transfer matrix element $R_{56}$ equaling zero. Usually sextupoles are needed to correct second-order dispersion in the bending plane, for both the dogleg optics and the chicane (with quad) optics. In this paper, an alternative optics design is introduced, which is based on a simple FODO cell and does not need sextupole assistance to form a second-order achromat. It may provide a similar function of either a dogleg or a bypass, by using two or four of such combined supercells.
\end{abstract}

DOI: 10.1103/PhysRevSTAB.14.060703

PACS numbers: 29.20.db, 29.27.Bd

\section{OVERVIEW}

A transverse dogleg beam line or a bypass beam line is needed for many accelerator systems from the requirement on shifting the beam axis in some region, which is sometimes due to realistic constraints. Some of these constraints are: existing infrastructure on the original beam axis; upgrade of an old accelerator; multifunction linac which feeds several downstream beam lines, and so on. A special example which has wide applications is a free electron laser driving linac. At the end of such an FEL linac, the electron beam can be used to feed several different undulators to generate photons for different purposes. There are also other systems which make use of the dispersion generated in the bending plane to do a momentum collimation on the beam and to remove the halo particles. In the 1970s, Brown developed a systematic approach to design secondorder achromats from investigating matrices formulas [1], which uses at least four identical cells with dipoles, quadrupoles, and sextupoles and can eliminate all geometric and chromatic terms up to second order. Then Wan developed another approach with Lie algebra to design achromat to arbitrary order, taking advantage of the midplane symmetry and using multipole magnets for each order (for example, octupoles for third-order achromat) [2]. Usually these systems are designed with dipoles, quadrupoles, and sextupoles, to zero both first-order and second-order dispersion in the bending plane, and to provide an isochronous transfer system in the bypass case. Generally the use of

\footnotetext{
*yisun@slac.stanford.edu
}

Published by the American Physical Society under the terms of the Creative Commons Attribution 3.0 License. Further distribution of this work must maintain attribution to the author(s) and the published article's title, journal citation, and DOI. sextupole magnets implies a strict tolerance on the beam line alignments.

At the same time, in many of these beam lines, the electron beam is transported with a large energy spread which is in the percent level. To transport such a beam through the dogleg (or bypass) and preserve the transverse emittance at the same time is a difficult task. In the following sections, a FODO cell based optics design is described which eliminates the use of sextupoles. In one supercell, both the first-order $\left(R_{16}\right.$ and $\left.R_{26}\right)$ and second-order dispersion $\left(T_{166}\right.$ and $\left.T_{266}\right)$ terms are closed, which guarantees no chromatic emittance growth as the beam transports through this section. Usually the initial transverse beam emittance is small in such systems being considered and one can neglect the small impact of the nonzero geometrical terms. Macroparticle tracking simulations have been performed in the code ELEGANT [3], to evaluate the property of this optics design.

\section{EQUATION OF MOTION AND MATRIX TREATMENT}

A moving coordinate system originated on the central trajectory of the beam line is employed here. Each particle in the beam has coordinates of $\left(x, x^{\prime}, y, y^{\prime}, z, \delta_{p}\right)$. The divergences $x^{\prime}$ and $y^{\prime}$ are defined as the ratio of transverse momentum to longitudinal momentum. Without any approximations, the vector differential equation of motion is

$$
\frac{d^{2} \mathbf{T}}{d T^{2}}=\frac{q}{p} \frac{d \mathbf{T}}{d T} \times \mathbf{B}
$$

where $\mathbf{T}$ denotes the position vector of any particle moving along its trajectory, $T$ the distance traveled, $q$ the particle charge, and $\mathbf{B}$ the magnetic field.

With magnetic field expansion in three directions, the linearized (first-order) differential equation of motion for the transverse coordinates $x$ and $y$ is [4] 


$$
\begin{gathered}
x^{\prime \prime}+(1-n) h^{2} x=h \delta \\
y^{\prime \prime}+n h^{2} y=0,
\end{gathered}
$$

where $\delta=\Delta p / p_{0}$ denotes the relative momentum deviation from the on momentum particle, $h=B_{y}(0,0, t) / B \rho$, and the dimensionless parameter $n$ given in terms of the vertical magnetic field component as shown in the following formula:

$$
n=-\frac{1}{h B_{y}} \frac{\partial B_{y}}{\partial x}{ }_{x=0, y=0} .
$$

Consider only the linear (first-order) effect, after passing by a beam line, the final coordinates of any particle can be expressed by a linear function of its initial coordinates, as illustrated in the following formula:

$$
x_{1}=\left(x \mid x_{0}\right) x_{0}+\left(x \mid x_{0}^{\prime}\right) x_{0}^{\prime}+(x \mid \delta) \delta,
$$

where $\left(x \mid x_{0}\right)$ denotes the coefficient of $x_{0}$ in the equation for $x_{1}$, which is $R_{11}$ in the following matrix formula.

Regarding the longitudinal motion, the path length difference depends on the initial coordinates $\left(x, x^{\prime}, y\right.$, $\left.y^{\prime}, z, \delta_{p}\right)$. From the vector differential equation of motion, the difference in path length of an arbitrary particle is calculated as an integral [4]:

$$
l=\int_{0}^{t}\left(\left[\left(\frac{d x}{d \tau}\right)^{2}+\left(\frac{d y}{d \tau}\right)^{2}+(1+h x)^{2}\right]^{1 / 2}-1\right) d \tau .
$$

The transport of the particle's coordinates can be treated in a general matrix style as shown below:

$$
\begin{aligned}
\left(\begin{array}{l}
x \\
x^{\prime} \\
y \\
y^{\prime} \\
z \\
\delta
\end{array}\right)= & \mathbf{R} \cdot\left(\begin{array}{l}
x_{0} \\
x_{0}^{\prime} \\
y_{0} \\
y_{0}^{\prime} \\
z_{0} \\
\delta_{0}
\end{array}\right) \\
= & \left(\begin{array}{llllll}
R_{11} & R_{12} & R_{13} & R_{14} & R_{15} & R_{16} \\
R_{21} & R_{22} & R_{23} & R_{24} & R_{25} & R_{26} \\
R_{31} & R_{32} & R_{33} & R_{34} & R_{35} & R_{36} \\
R_{41} & R_{42} & R_{43} & R_{44} & R_{45} & R_{46} \\
R_{51} & R_{52} & R_{53} & R_{54} & R_{55} & R_{56} \\
R_{61} & R_{62} & R_{63} & R_{64} & R_{65} & R_{66}
\end{array}\right)\left(\begin{array}{c}
x_{0} \\
x_{0}^{\prime} \\
y_{0} \\
y_{0}^{\prime} \\
z_{0} \\
\delta_{0}
\end{array}\right),
\end{aligned}
$$

where $\mathbf{R}$ denotes the first-order transport matrix of a beam line.

Given the magnetic field of a specified type of element, such as a dipole magnet, one can solve the linearized equation of motion and get the transfer matrix $\mathbf{R}$. Shown below are the first-order transfer matrices of a dipole, a quadrupole, a sextupole, and a drift, under thin lens approximation $[4,5]$. Note that the discussion here is under the ultrarelativistic condition.

The six-by-six transport matrix of the normal dipole magnet with bending angle $\theta$ and bending radius $\rho$ can be written as

$$
\begin{aligned}
& \mathbf{R}_{\mathbf{B}(\theta, \rho)} \\
& =\left(\begin{array}{cccccc}
\cos \theta & \rho \sin \theta & 0 & 0 & 0 & \rho(1-\cos \theta) \\
-\sin \theta / \rho & \cos \theta & 0 & 0 & 0 & \sin \theta \\
0 & 0 & 1 & \rho \theta & 0 & 0 \\
0 & 0 & 0 & 1 & 0 & 0 \\
-\sin \theta & \rho(\cos \theta-1) & 0 & 0 & 1 & \rho(\sin \theta-\theta) \\
0 & 0 & 0 & 0 & 0 & 1
\end{array}\right) .
\end{aligned}
$$

The six-by-six transport matrix of a quadrupole magnet with integrated strength $K_{1}$ can be written as

$$
\mathbf{R}_{\text {quad }}=\left(\begin{array}{cccccc}
1 & L_{Q} & 0 & 0 & 0 & 0 \\
-K_{1} & 1 & 0 & 0 & 0 & 0 \\
0 & 0 & 1 & L_{Q} & 0 & 0 \\
0 & 0 & K_{1} & 1 & 0 & 0 \\
0 & 0 & 0 & 0 & 1 & 0 \\
0 & 0 & 0 & 0 & 0 & 1
\end{array}\right) .
$$

The six-by-six transport matrix of a drift space with length $L$ can be written as

$$
\mathbf{R}_{\text {drift }}=\left(\begin{array}{cccccc}
1 & L & 0 & 0 & 0 & 0 \\
0 & 1 & 0 & 0 & 0 & 0 \\
0 & 0 & 1 & L & 0 & 0 \\
0 & 0 & 0 & 1 & 0 & 0 \\
0 & 0 & 0 & 0 & 1 & 0 \\
0 & 0 & 0 & 0 & 0 & 1
\end{array}\right) .
$$

A sextupole is a type of second-order magnetic element which does not change the first-order optics. Its first-order transport matrix is the same with a drift space of the same length.

For monochromatic beams which occupy a very small phase space, a first-order transport matrix is a good approximation to describe the transmission properties of a beam line. For a realistic beam which usually has large energy spread, second-order and higher order aberrations have to be taken into consideration. A general matrix notation for the transport of particles' coordinates is shown in the following formula, which has both first-order and second-order terms [4]:

$$
x_{i}(1)=\sum_{j} R_{i j} x_{j}(0)+\sum_{j, k} T_{i j k} x_{j}(0) x_{k}(0),
$$


where second-order matrix $T$ has entries of the quadratic terms in the expansion of the final coordinates as a function of the initial coordinates.

To derive the second-order matrix elements for individual magnets, as has been done for the first-order matrix, one needs to again solve the equation of motion up to second order. Take a second-order expansion in the transverse coordinates, and apply magnetic field expansion, the equation of motion is (rectangular coordinate system) [4]

$$
\begin{aligned}
x^{\prime \prime}+(1-n) h^{2} x= & h \delta+(2 n-1-\beta) h^{3} x^{2}+h^{\prime} x x^{\prime}+\frac{1}{2} h x^{\prime 2} \\
& +(2-n) h^{2} x \delta+\frac{1}{2}\left(h^{\prime \prime}-n h^{3}+2 \beta h^{3}\right) y^{2} \\
& +h^{\prime} y y^{\prime}-\frac{1}{2} h y^{\prime 2}-h \delta^{2}
\end{aligned}
$$

$y^{\prime \prime}+n h^{2} y=2(\beta-n) h^{3} x y+h^{\prime} x y^{\prime}-h^{\prime} x^{\prime} y+h x^{\prime} y^{\prime}+n h^{2} y \delta$.

A general solution of the above two differential equations consists of the solution of the homogeneous equation, and a particular solution of the inhomogeneous equation. The second-order matrix element can be calculated as the Green's function integral of the associated driving term. The path length difference can be calculated using the same integration while retaining terms up to second order. Now, the same as the calculation of the $\mathbf{R}$ (first-order) matrices elements, one can calculate all the $\mathbf{T}$ (secondorder) matrices elements, given the magnetic field of a specified type of element, such as a quadrupole magnet. As discussed before, a drift space has no second-order effects on the transport of particles' transverse coordinates. However, a drift does have two nonzero longitudinal $\mathbf{T}$ (second-order) matrices elements, $T_{522, D}=T_{544, D}=$ $-\frac{1}{2} L_{D}$. For a quadrupole magnet with integrated strength $K_{1}$ and length $L_{Q}$, its nonzero second-order matrices elements are listed below:

$$
\begin{gathered}
T_{116, Q}=\frac{1}{2} K_{1} L_{Q}, \quad T_{126, Q}=-\frac{1}{2} L_{Q}, \\
T_{216, Q}=K_{1}, \quad T_{226, Q}=\frac{1}{2} K_{1} L_{Q} \\
T_{336, Q}=-\frac{1}{2} K_{1} L_{Q}, \quad T_{346, Q}=-\frac{1}{2} L_{Q}, \\
T_{436, Q}=-K_{1}, \quad T_{446, Q}=-\frac{1}{2} K_{1} L_{Q} \\
T_{512, Q}=\frac{1}{2} K_{1} L_{Q}, \quad T_{522, Q}=-\frac{1}{2} L_{Q}, \\
T_{534, Q}=-\frac{1}{2} K_{1} L_{Q}, \quad T_{544, Q}=-\frac{1}{2} L_{Q} .
\end{gathered}
$$

For a sextupole magnet with integrated strength $K_{2}$ and length $L_{S}$, its nonzero second-order matrices elements are listed below:

$$
\begin{gathered}
T_{111, S}=-\frac{1}{2} K_{2} L_{S}, \quad T_{112, S}=-\frac{1}{3} K_{2} L_{S}^{2}, \\
T_{122, S}=-\frac{1}{12} K_{2} L_{S}^{3}
\end{gathered}
$$

$$
\begin{gathered}
T_{133, S}=\frac{1}{2} K_{2} L_{S}, \quad T_{134, S}=\frac{1}{3} K_{2} L_{S}^{2}, \quad T_{144, S}=\frac{1}{12} K_{2} L_{S}^{3} \\
T_{211, S}=-K_{2}, \quad T_{212, S}=-K_{2} L_{S}, \quad T_{222, S}=-\frac{1}{3} K_{2} L_{S}^{2} \\
T_{233, S}=K_{2}, \quad T_{234, S}=K_{2} L_{S}, \quad T_{244, S}=\frac{1}{3} K_{2} L_{S}^{2} \\
T_{313, S}=K_{2} L_{S}, \quad T_{314, S}=\frac{1}{3} K_{2} L_{S}^{2} \\
T_{323, S}=\frac{1}{3} K_{2} L_{S}^{2}, \quad T_{324, S}=\frac{1}{6} K_{2} L_{S}^{3} \\
T_{413, S}=2 K_{2}, \quad T_{414, S}=K_{2} L_{S} \\
T_{423, S}=K_{2} L_{S}, \quad T_{424, S}=\frac{2}{3} K_{2} L_{S}^{2} \\
T_{522, S}=-\frac{1}{2} L_{S}, \quad T_{544, S}=-\frac{1}{2} L_{S}
\end{gathered}
$$

For a dipole magnet with bending angle $\theta$ and bending radius $\rho$, its critical second-order matrices elements are listed below:

$$
\begin{aligned}
& T_{111, B}=-\frac{1}{2 \rho} \sin ^{2} \theta, \quad T_{112, B}=\sin \theta \cos \theta, \\
& T_{122, B}=\frac{1}{2} \rho \cos \theta(1-\cos \theta) \\
& T_{133, B}=0, \quad T_{134, B}=0, \quad T_{144, B}=-\frac{1}{2} \rho(1-\cos \theta) \\
& T_{116, B}=\sin ^{2} \theta, \quad T_{126, B}=\rho \sin \theta(1-\cos \theta), \\
& T_{166, B}=-\frac{1}{2} \rho \sin ^{2} \theta \\
& T_{211, B}=0, \quad T_{212, B}=0, \quad T_{222, B}=-\frac{1}{2} \sin \theta \\
& T_{233, B}=0, \quad T_{234, B}=0, \quad T_{244, B}=-\frac{1}{2} \sin \theta \\
& T_{216, B}=\frac{\sin \theta}{\rho}, \quad T_{226, B}=0, \quad T_{266, B}=-\sin \theta \\
& T_{313, B}=0, \quad T_{314, B}=\sin \theta, \quad T_{323, B}=0, \\
& T_{324, B}=\rho(1-\cos \theta), \quad T_{336, B}=0, \\
& T_{346, B}=\rho \theta-\rho \sin \theta \\
& T_{413, B}=0, \quad T_{414, B}=0, \quad T_{423, B}=0, \\
& T_{424, B}=0, \quad T_{436, B}=0, \quad T_{446, B}=0 \\
& T_{511, B}=0, \quad T_{512, B}=0, \quad T_{522, B}=-\frac{1}{2} \rho \sin \theta \\
& T_{533, B}=0, \quad T_{534, B}=0, \quad T_{544, B}=-\frac{1}{2} \rho \sin \theta \\
& T_{516, B}=0, \quad T_{526, B}=-\rho(1-\cos \theta), \quad T_{566, B}=0 . \\
&
\end{aligned}
$$


Given known first-order and second-order transport matrices of all the magnetic elements in a lattice, one could multiply them subsequently to get the transport matrix of the whole system. As an example, for a lattice which consists of a dipole magnet, a drift, and a quadrupole magnet, the overall transfer matrix reads (note the sequence of the multiplication)

$$
\begin{aligned}
& \mathbf{R}_{\text {all }}=\mathbf{R}_{\text {quad }} \cdot \mathbf{R}_{\text {drift }} \cdot \mathbf{R}_{\mathbf{B}(\theta, \rho)} \\
& \mathbf{T}_{\text {all }}=\mathbf{T}_{\text {quad }} \cdot \mathbf{T}_{\text {drift }} \cdot \mathbf{T}_{\mathbf{B}(\theta, \rho)} .
\end{aligned}
$$

To transport the particle's coordinates through a beam line using an up to second-order matrices approach, in general one need a combined overall transport matrix which has 42-by-42 entries, which is reduced to 27 -by-27 entries neglecting all the duplicated terms. In a simplified form (neglect all the zero and duplicated terms), the transport of the particle's coordinates in the bending plane is (up to second order)

$$
\left(\begin{array}{c}
x \\
x^{\prime} \\
z \\
\delta \\
x^{2} \\
x x^{\prime} \\
x z \\
x \delta \\
x^{\prime 2} \\
x^{\prime} z \\
x^{\prime} \delta \\
z^{2} \\
z \delta \\
\delta^{2} \\
y^{2} \\
y y^{\prime} \\
y^{\prime 2}
\end{array}\right)=\mathbf{T}_{\mathbf{x},[17 \times \mathbf{1 7}]} \cdot\left(\begin{array}{c}
x_{0} \\
x_{0}^{\prime} \\
z_{0} \\
\delta_{0} \\
x_{0}^{2} \\
x_{0} x_{0}^{\prime} \\
x_{0} z_{0} \\
x_{0} \delta_{0} \\
x_{0}^{\prime 2} \\
x_{0}^{\prime} z_{0} \\
x_{0}^{\prime} \delta_{0} \\
z_{0}^{2} \\
z_{0} \delta_{0} \\
\delta_{0}^{2} \\
y_{0}^{2} \\
y_{0} y_{0}^{\prime} \\
y_{0}^{\prime 2}
\end{array}\right) .
$$

The second-order transfer matrix in the bending plane can be expressed in the submatrix form of

$\mathbf{T}_{\mathrm{x},[17 \times 17]}=\left(\begin{array}{ccc}\mathbf{T}_{11,[4 \times 4]} & \mathbf{T}_{12,[4 \times 10]} & \mathbf{T}_{13,[4 \times 3]} \\ \mathbf{0}_{[10 \times 4]} & \mathbf{T}_{22,[10 \times 10]} & \mathbf{0}_{[10 \times 3]} \\ \mathbf{0}_{[3 \times 4]} & \mathbf{0}_{[3 \times 10]} & \mathbf{T}_{33,[3 \times 3]}\end{array}\right)$,

where $\mathbf{0}_{[\mathbf{3} \times \mathbf{6}]}$ denotes a three-by-six zero matrix, $\mathbf{T}_{\mathbf{2 2},[\mathbf{1 0} \times \mathbf{1 0}]}$ a ten-by-ten matrix, and all five submatrices are listed in the Appendix. The transport of the particle's coordinates in the nonbending plane is much easier and not discussed in detail here.

\section{FIRST-ORDER OPTICS}

In this section, a standard FODO lattice is studied, to investigate the first-order achromat condition and the associated relation between parameters of different magnets. A schematic plot of one super period which is composed of four identical standard FODO cells is shown in Fig. 1. One FODO cell starts from a half focusing quadrupole with integrated strength $K_{1 f}$, plus a drift with length $l$, followed by a dipole magnet with bending angle $\theta$ and bending radius $\rho$, another drift with the same length, and a full defocusing quadrupole with integrated strength $2 K_{1 d}$. This FODO cell ends at another half focusing quadrupole magnet.

Applying the matrices approach discussed in the previous section, the overall first-order transport matrix of one FODO cell is

$$
\mathbf{R}_{\mathrm{FODO}}=\mathbf{R}_{\mathrm{Qfh}} \cdot \mathbf{R}_{\mathrm{D}} \cdot \mathbf{R}_{\mathbf{B}} \cdot \mathbf{R}_{\mathbf{D}} \cdot \mathbf{R}_{\mathrm{QD}} \cdot \mathbf{R}_{\mathbf{D}} \cdot \mathbf{R}_{\mathrm{B}} \cdot \mathbf{R}_{\mathbf{D}} \cdot \mathbf{R}_{\mathrm{Qfh}} \cdot
$$

Under some simplification and thin lens approximation, the six-by-six transport matrix $\mathbf{R}_{\mathrm{FODO}}$ of this FODO cell can be calculated. Here, in particular, three entries which are the dispersion terms are listed below:

$$
\begin{gathered}
\mathbf{R}_{16, \mathrm{FODO}}=\left[-4 K_{1 d} l^{2}+\left(4-2 K_{1 d} \rho \theta\right) l+\rho \theta\right] \theta \\
\mathbf{R}_{26, \mathrm{FODO}}=\left[4 K_{1 d} K_{1 f} l^{2}+\left(2 K_{1 d} K_{1 f} \rho \theta-4 K_{1 f}-2 K_{1 d}\right) l\right. \\
\left.-K_{1 f} \rho \theta+2\right] \theta \\
\mathbf{R}_{56, \mathrm{FODO}}=2\left(K_{1 d} l^{2}-l\right) \theta^{2} .
\end{gathered}
$$

One needs to note that $\rho, \theta$, and $K_{1 f}$ are all positive, and $K_{1 d}$ is negative. Given these conditions, it is obvious to observe that for such a FODO cell: the first-order dispersion term $\mathbf{R}_{16, \text { FODO }}$ is always positive; the value of the firstorder angular dispersion term $\mathbf{R}_{26, \text { FODO }}$ depends on the choice of $\rho, \theta, l, K_{1 d}$, and $K_{1 f}$, which can be either positive or negative; the longitudinal first-order dispersion term $\mathbf{R}_{56, \mathrm{FODO}}$ is always negative.

To illustrate the relations between the dispersion terms and the magnet parameters, here we fix some of the magnet parameters and plot the dispersion terms as a function of $K_{1 d}$, as shown in Fig. 2. For the dipole magnet, the bending angle is chosen to be $2.5^{\circ}$ with a bending radius of $\rho=$ $4.6 \mathrm{~m}$. The integrated strength of the half focusing quadrupole is $K_{1 f}=1.6$, with a half effective magnetic length of $0.1 \mathrm{~m}$. The drift space length is $l=0.25 \mathrm{~m}$. As illustrated in Fig. 2, for a larger absolute value of $K_{1 d}$, the absolute values of $\mathbf{R}_{16 \text {,FODO }}$ and $\mathbf{R}_{56 \text {,FODO }}$ are both larger. At the same time, for given $\rho, \theta, l, K_{1 d}$, and $K_{1 f}$, there is a unique defocusing quadrupole strength $K_{1 d, 0}$, to zero the first-order angular dispersion $\mathbf{R}_{26 \text {,FODO }}$ at the end of this FODO cell. 


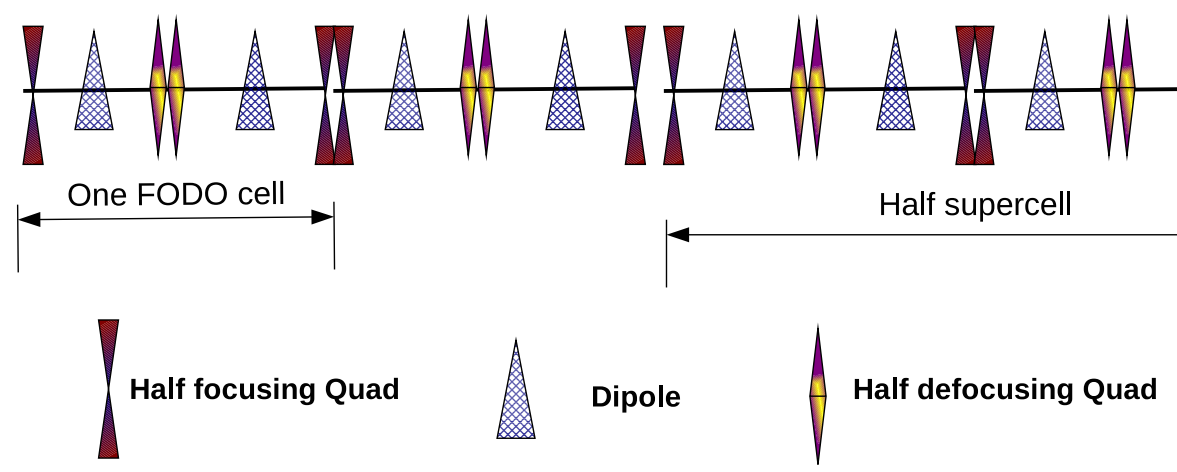

FIG. 1. Sketch of one super period of the optics under study.

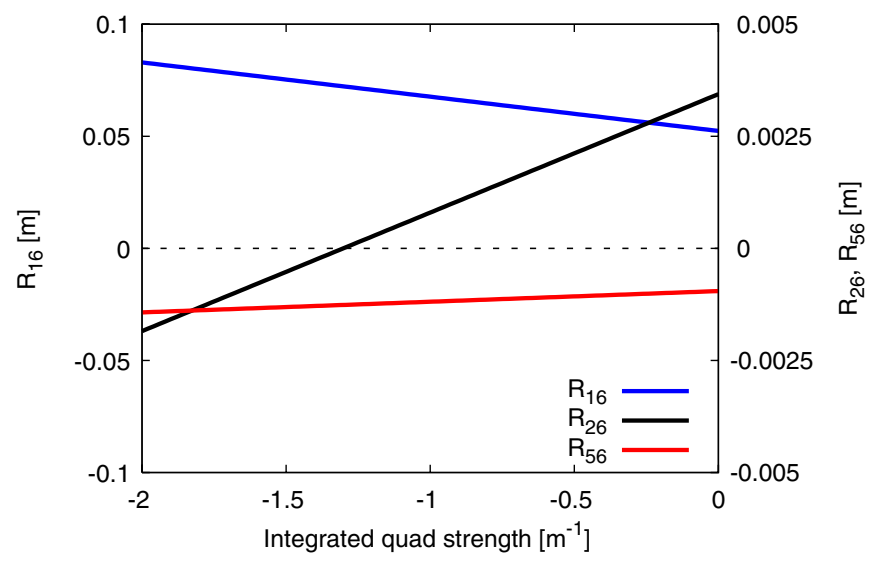

FIG. 2. Dispersion terms $\mathbf{R}_{16, \text { FODO }}, \mathbf{R}_{26, \text { FODO }}$, and $\mathbf{R}_{56 \text {,FODO }}$ of one FODO arc cell, versus the integrated strength of the defocusing quadrupole $K_{1 d}$.

To achieve a first-order achromat $\left(\mathbf{R}_{16}=\mathbf{R}_{26}=0\right)$ in one supercell as shown in Fig. 1, the angular dispersion term $\mathbf{R}_{26}$ has to be zero at the middle point, due to symmetry requirement. This condition can be approximately extended to a zero angular dispersion at the end of the first FODO cell, which is the middle point of the half supercell. One can then solve the equation and express the required defocusing quadrupole strength as a function of $\rho$, $\theta, l$, and $K_{1 f}$, as shown below:

$$
K_{1 d}=\frac{4 K_{1 f} l+K_{1 f} \rho \theta-2}{4 K_{1 f} l^{2}+\left(2 K_{1 f} \rho \theta-2\right) l} .
$$

One can calculate the first-order transport matrix of a half supercell as shown below, and get a precise solution of $K_{1 d, 0}$. That is much more complicated and is not discussed in detail here:

$$
\mathbf{R}_{\text {half, supercell }}=\mathbf{R}_{\mathrm{FODO}} \cdot \mathbf{R}_{\mathrm{FODO}} .
$$

In designing a lattice, one also needs to constrain the amplitude of the betatron function $\beta$ in a proper range, which has a maximum and a minimum value depending on the cell length and quadrupole strength (for a standard FODO cell), as shown below:

$$
\beta \pm=\frac{2\left(1 \pm \frac{L_{\text {cell }}}{4} K_{1}\right)}{K_{1} \sqrt{1-\left(\frac{L_{\text {cell }}}{4} K_{1}\right)^{2}}},
$$

where $\beta \pm$ denotes the maximum and minimum beta functions, $L_{\text {cell }}$ the FODO cell length, and $K_{1}$ the integrated quadrupole strength.

Under the following considerations, an optics is designed as sketched in Fig. 1. A dipole magnet with a length of $0.2 \mathrm{~m}$ and a bending angle of $2.5^{\circ}$ is chosen. The drift space length is $l=0.15 \mathrm{~m}$ for thick quadrupoles whose length is $0.2 \mathrm{~m}$. The variation range of the beta functions is constrained between 0.5 and $10 \mathrm{~m}$. The quadrupole strengths $K_{1 d}$ and $K_{1 f}$ are estimated by using formulas (43) and (45), and further optimized by matching in the accelerator design code MAD8 [6]. After optimization, the first-order Twiss parameters are shown in Fig. 3, where a first-order achromat condition is achieved.

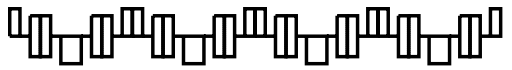

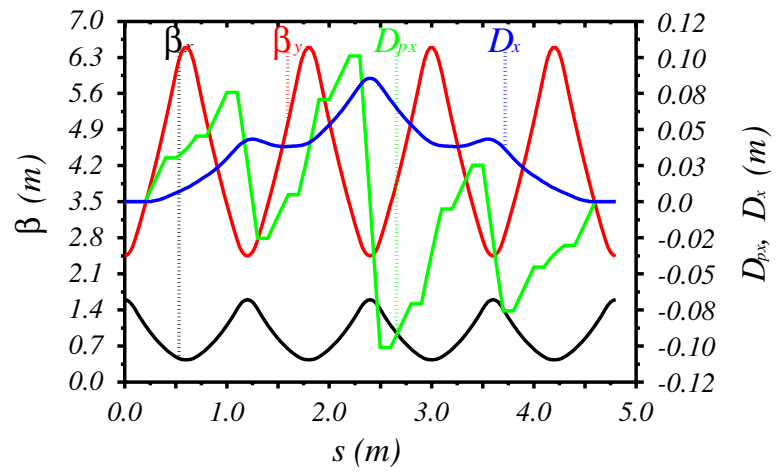

FIG. 3. First order dispersion and beta functions of one supercell which consists of four identical FODO cells: black curve denotes horizontal beta function, red curve denotes vertical beta function, blue curve denotes horizontal dispersion function, and green curve denotes horizontal angular dispersion function. The color notation is the same for the first-order optics in the following figures. 
In order to make this supercell extendable, the beta functions need to be identical at the entrance and at the exit. At the same time, the alpha function which is proportional to the derivative of the beta function needs to equal zero. The second-order horizontal dispersion terms $T_{166}$ and $T_{266}$ are not zero under these constraints. One can then add sextupoles in the optics and zero the second-order dispersion.

\section{SECOND-ORDER OPTICS}

As discussed above, the second-order transport matrices of a half supercell can be calculated as in the following formula, which is the multiplication of two identical FODO cell matrices. The second-order transport matrix of one FODO cell is the multiplication of the matrices of its subsequent elements, also shown below:

$$
\begin{gathered}
\mathbf{T}_{\text {half,supercell }}=\mathbf{T}_{\mathrm{FODO}} \cdot \mathbf{T}_{\mathrm{FODO}} \\
\mathbf{T}_{\mathrm{FODO}}=\mathbf{T}_{\mathbf{Q f h}} \cdot \mathbf{T}_{\mathbf{D}} \cdot \mathbf{T}_{\mathbf{B}} \cdot \mathbf{T}_{\mathbf{D}} \cdot \mathbf{T}_{\mathbf{Q D}} \cdot \mathbf{T}_{\mathbf{D}} \cdot \mathbf{T}_{\mathbf{B}} \cdot \mathbf{T}_{\mathbf{D}} \cdot \mathbf{T}_{\mathbf{Q f h}} \cdot
\end{gathered}
$$

Now one can get the expression of the second-order dispersion $T_{166}$ and the angular dispersion $T_{266}$, which are all functions of $\rho, \theta, l, K_{1 d}$, and $K_{1 f}$ (still under thin lens approximation). The detailed expression of $T_{166}$ and $T_{266}$ are more complicated than $\mathbf{R}_{16}$ or $\mathbf{R}_{26}$, so not listed here for simplicity. Similarly as the first-order optics, to achieve a second-order achromat $\left(\mathbf{T}_{166}=\mathbf{T}_{266}=0\right)$ in one supercell as shown in Fig. 1, the angular dispersion term $\mathbf{T}_{266}$ has to be zero at the middle point, due to symmetry requirement. For a supercell which consists of four identical FODO cells, this requirement is not easy to fulfill.

However, through some analytical and numerical manipulations, one finds that by adopting the missing dipole option, it is possible to get a second-order achromat without the assistance of sextupoles. In the analytical manipulations, a small angle approximation is adopted for the dipole magnet model, and one then has $\sin \theta \approx \theta$ and $\cos \theta \approx 1$. With this treatment, the expression of the second-order dispersions $T_{166}$ and $T_{266}$ is simplified and much easier to manipulate. In general, a second-order achromatic condition is solved by finding parameter relations which fulfill $\mathbf{T}_{266 \text {,mid }}=0$ at the middle point of the supercell, and $\mathbf{T}_{166 \text {, end }}=0$ at the end of the supercell, plus the first-order achromat condition $\mathbf{R}_{26 \text {,mid }}=0$ and $\mathbf{R}_{16, \text { end }}=0$. These four expressions $\left(\mathbf{R}_{26, \text { mid }}, \mathbf{R}_{16, \text { end }}\right.$, $\mathbf{T}_{266, \text { mid }}$, and $\mathbf{T}_{166, \text { end }}$ ) are all functions of $\rho, \theta, l, K_{1 d}$, and $K_{1 f}$ (under thin lens approximation). The four zero conditions of the dispersion terms are manipulated analytically using a computer algebra system in MAXIMA [7]. As discussed in detail below, one could find reasonable solutions of $\rho, \theta_{1}\left(\theta_{2}\right), l, K_{1 d}$, and $K_{1 f}$ under the missing dipole configuration. The solution is unique if two of the six variables are fixed. Then these estimated magnet and drift parameters are further matched and optimized in the accelerator design code MAD8 [6], by setting a proper matching constraint (such as $\mathbf{T}_{266}=0$ at the middle, $\mathbf{T}_{166}=0$ at the end, reasonable beta functions plus the first-order dispersion requirements). The variables $\rho, \theta_{1}\left(\theta_{2}\right), l, K_{1 d}$, and $K_{1 f}$ are all constrained in a reasonable range.

Under the missing dipole configuration, the second and the seventh dipole are removed in the supercell while the other six dipoles are kept. By slightly modifying the drift length, quadrupole strength, and also the periodic Twiss parameters, one can easily get a first-order achromat, as shown in Fig. 4. The first-order dispersion oscillation along this beam line is different from the one in Fig. 3, due to the impact from two missing dipoles. The second-order dispersion terms $T_{166}$ and $T_{266}$ are much smaller at the end than the previous case, but still not zero.

One possible second-order achromat condition is achieved by further slightly adjusting the bending angle of the first and last dipole magnets. At the same time, the quadrupole strength and drift length are also slightly tuned, with the constraints that both first-order and second-order dispersion terms are zero $\left(\mathbf{R}_{16}=\mathbf{R}_{26}=0\right.$ and $\mathbf{T}_{166}=\mathbf{T}_{266}=0$ ). That optimization changes the evolution of the second-order dispersion and keeps the first-order dispersion oscillation similar with the same dipole strength case. The final bending angle of the first and the last dipole is chosen to be $1.65^{\circ}$ each, and it is $1.72^{\circ}$ each for the other four dipoles in the center two FODO cells. The drift length is minimized and a final length of $0.15 \mathrm{~m}$ is achieved. The quadrupole strength is also minimized and the final choice is an integrated strength of -1.6 and +2.6 , respectively. The first-order optics is almost the same as the one shown in Fig. 4. The maximum beta function $\beta_{y}$ and dispersion function $D_{x}$ are 6 and $0.04 \mathrm{~m}$, respectively.
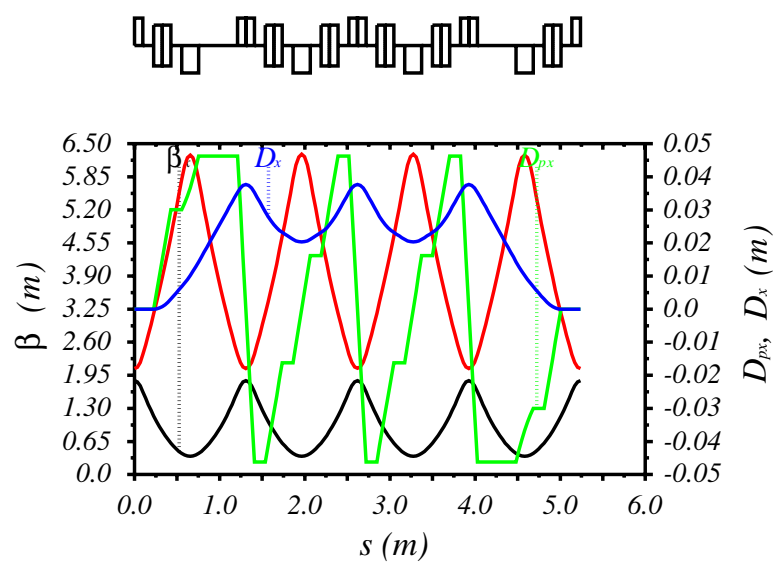

FIG. 4. First order dispersion and beta functions of one supercell which consists of four FODO cells, under the missing dipole configuration. 

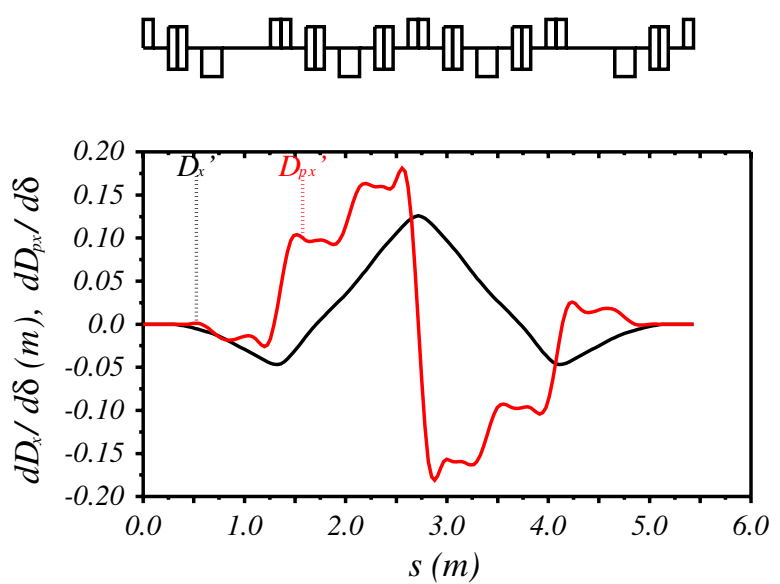

FIG. 5. Second-order dispersion $T_{166}$ and angular dispersion $T_{266}$ of one supercell which consists of four FODO cells, under the missing dipole configuration.

The evolution of the second-order dispersion $T_{166}$ and $T_{266}$ is illustrated in Fig. 5, where one observes that the middle point symmetry is always maintained. The maximum absolute value of the second-order dispersion is $0.17 \mathrm{~m}$ which is relatively small. Notice that for this design either the focusing quadrupoles or the defocusing quadrupoles have the same strength. Here we note that there are also other possible manipulations of this supercell, to achieve a second-order achromat, except the missing dipole configuration described here.

In the following section, two application examples using this supercell design are presented. One is a dogleg beam line design, while the other is a bypass beam line design. Both of these two beam lines are isochronous $\left(R_{56}=0\right)$.

\section{POSSIBLE APPLICATIONS}

As discussed above, the beta functions $\beta_{x}$ and $\beta_{y}$ are symmetric at the two ends of this supercell, and the beta function derivative $\alpha$ equals zero. That feature makes this supercell easy to be extended. Two possible applications are described here, with their sketch shown in Fig. 6. In the dogleg beam line, there is one normal supercell followed by a second supercell in which all six dipole magnets switch sign and bend the beam to the opposite direction. This turns the direction of the beam line back (there is an angle due to the first supercell) to the original one, but generates an offset in the bending plane. Using the magnet parameters as specified in the above section, one can do the calculation (matching) and find that each supercell has a first-order longitudinal dispersion $R_{56}=2.5 \mathrm{~mm}$. One can then add a small three (four) dipole chicane after the second supercell, which generates a longitudinal dispersion $R_{56}=-5 \mathrm{~mm}$ and makes the overall system isochronous. There is a small second-order longitudinal dispersion $T_{566}$ left on the system which effect should be negligible.

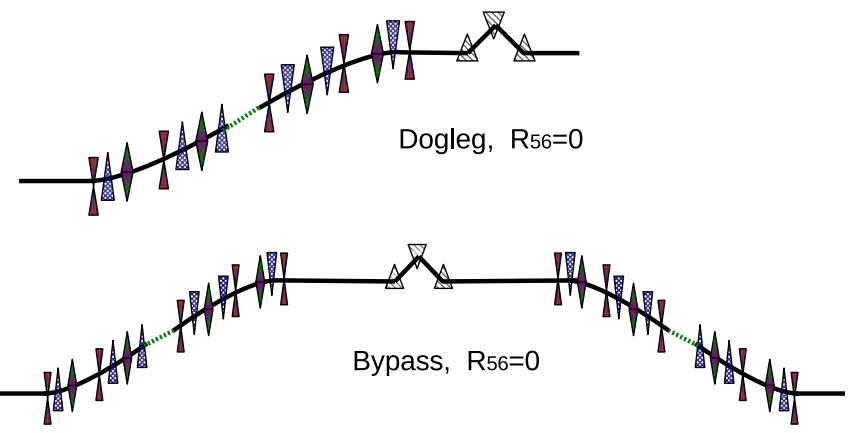

FIG. 6. Sketch of a dogleg beam line and a bypass beam line, $R_{56}=0$ in both cases.

The lower plot in Fig. 6 is one possible design of a bypass using these supercells, where one needs to shift the beam line with an offset in the bending plane first, then shift it back to the original beam line. Four supercells are employed here to construct this bypass. One may easily flip the two supercells in the dogleg (previous example) and add the flipped one after the small chicane which is in the middle. The middle small chicane also needs to be adjusted to provide 2 times longitudinal dispersion $R_{56}=-10 \mathrm{~mm}$, either by using a stronger dipole or by lengthening the drift length between the dipoles. The length of the straight section in the middle can be lengthened or shortened according to the real constraints, and one only need to add more quadrupoles if a longer straight section is necessary.

The first-order and second-order optics of these two beam lines are matched in MAD8 [6] and presented here in Fig. 7. The length of the dogleg beam line is $14 \mathrm{~m}$, which implies an offset of $1 \mathrm{~m}$ in the bending plane. The offset can be increased by lengthening the drift between the two supercells and one also needs to insert several quadrupoles to match the optics there. As mentioned above, the firstorder longitudinal dispersion $R_{56}=0$ and there is a small second-order dispersion $T_{566}$ left. The length of the bypass beam line is $30 \mathrm{~m}$ in all, where $8 \mathrm{~m}$ is devoted on the middle straight section. Similarly, the offset of $1 \mathrm{~m}$ in the bending plane can be tuned by adjusting the drift length. The length of the middle straight is also easy to tune, to accommodate different beam line elements in a real bypass. A middle plane symmetry is maintained in the bypass beam line and in the two supercells of the dogleg beam line, which is useful in canceling optics aberrations. One may also need to note that the betatron phase advance in the middle straight of the bypass beam line is important in a fine-tuning of the system.

The maximum beta function is $8 \mathrm{~m}$ in both beam lines, accompanied by a maximum dispersion $D_{x}=0.05 \mathrm{~m}$. The transport matrices elements $R_{56}$ and $T_{566}$, also the dipole and quadrupole parameters are listed in Table I below, assuming a beam energy of $1 \mathrm{GeV}$. The pole-tip field of the dipole and quadrupole magnets is calculated by using 


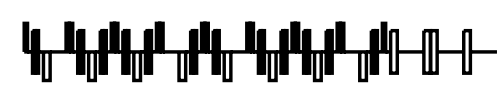

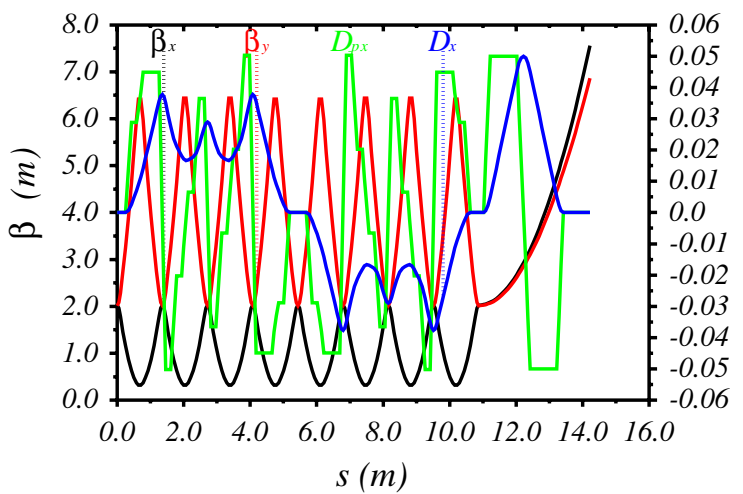

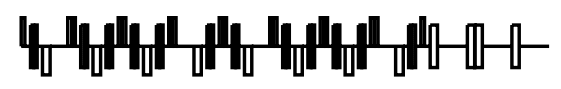

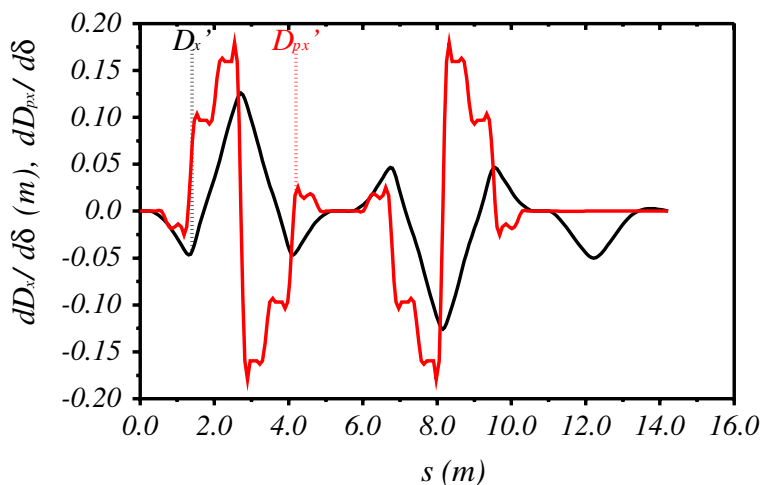

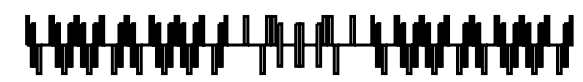

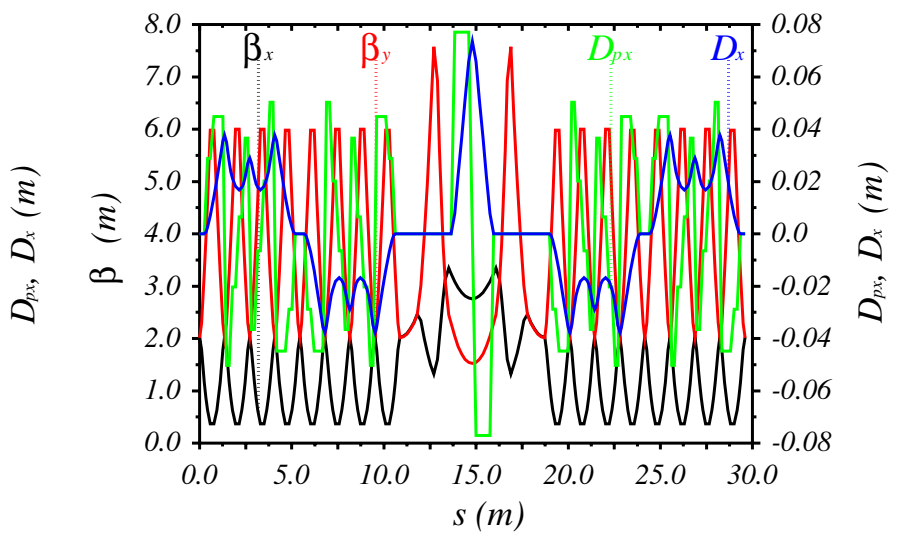

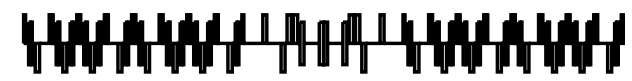

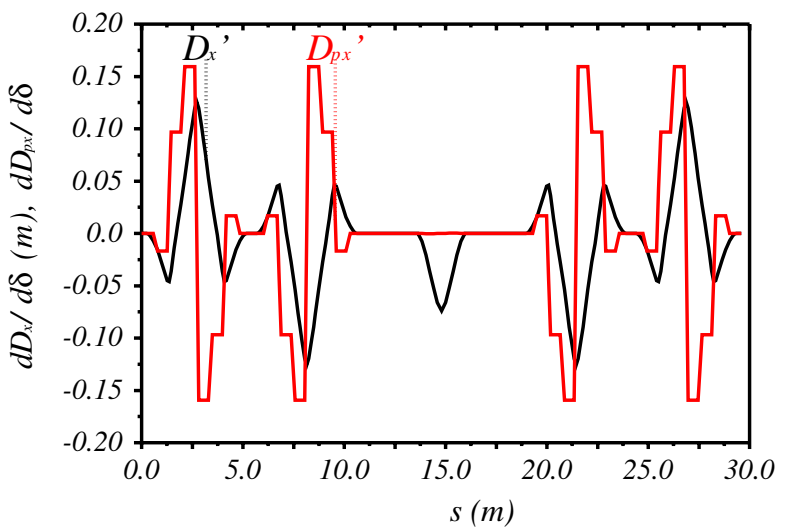

FIG. 7. First order and second-order optics of a dogleg beam line and a bypass beam line. Left top: First order dispersion and beta functions of the dogleg beam line. Left bottom: Second-order dispersion $T_{166}$ and angular dispersion $T_{266}$ of the dogleg beam line. Right top: First order dispersion and beta functions of the bypass beam line. Right bottom: Second-order dispersion $T_{166}$ and angular dispersion $T_{266}$ of the bypass beam line.

the following two formulas, where a half gap of $1.5 \mathrm{~cm}$ is assumed. Note that for higher beam energy one may need to increase the dipole magnet length (current length $0.2 \mathrm{~m}$ ) to maintain a weaker pole-tip field and partially suppress the incoherent synchrotron radiation effects:

$$
\begin{gathered}
B_{\text {dipole }}=\frac{B \rho \cdot \theta}{L_{\text {dipole }}} \\
B_{\text {quad }}=K_{1} \cdot B \rho \cdot a .
\end{gathered}
$$

\section{ELEGANT SIMULATION}

In order to evaluate the property of these two beam lines in transporting a real beam, the optics described in the above section is converted into the accelerator code ELEGANT [3]. First of all, the linear and second-order optics (all the transport matrices elements) are compared between MAD8 and ELEGANT, where a good agreement is achieved. Second, a beam which consists of $1 \times 10^{6}$ macroparticles is transported through these two beam lines. Then different beam parameters, such as projected normalized emittance, sliced normalized emittance, sliced energy spread, and the

TABLE I. Beam line parameters (at a beam energy of $1 \mathrm{GeV}$ ).

\begin{tabular}{lccccccc}
\hline \hline Name & $L[\mathrm{~m}]$ & Bend number & $\theta[\mathrm{d}]$ & $B_{\text {dipole }}[\mathrm{kG}]$ & $B_{\text {quad }}[\mathrm{kG}]$ & $R_{56}[\mathrm{~mm}]$ & $T_{566}[\mathrm{~mm}]$ \\
\hline Dogleg & 14 & 16 & 1.7 & 4.8 & 5.4 & 0 & 22 \\
Bypass & 30 & 28 & 1.7 & 4.8 & 5.4 & 0 & 47 \\
\hline \hline
\end{tabular}


longitudinal phase space shape are investigated. A beam of $1 \times 10^{6}$ macroparticles $\left(x, x^{\prime}, y, y^{\prime}, z, \delta_{p}\right)$ is generated in ELEGANT, with an rms normalized transverse emittance of $\gamma \epsilon_{x}=\gamma \epsilon_{y}=0.5 \mu \mathrm{m}$, an rms energy spread of $\sigma_{\delta_{p}}=$ $1 \%$, an rms bunch length of $\sigma_{z}=300 \mu \mathrm{m}$, and a beam energy of $1 \mathrm{GeV}$.

The simulation results of these two beam lines are very similar, and here only the results of the dogleg beam line is presented, for simplicity. The rms bunch length and projected normalized emittance evolution along the bypass beam line is shown in Fig. 8. From Fig. 8 (left), one observes that the rms bunch length changes along the beam line as the summed longitudinal dispersion $R_{56}$ changes. However, at the end of the bypass the initial bunch length is resumed due to a total dispersion $R_{56}=0$. With a relatively large energy spread of $\sigma_{\delta_{p}}=$ $1 \%$, this also demonstrates that the effect from small residual second-order dispersion $T_{566}$ is negligible. On the other hand, the projected normalized emittance is also preserved through the transport in this beam line, as shown in Fig. 8 (right). In the dispersive region between the dipole magnets, the large momentum offset couples into the projected emittance and makes it much larger.

Many accelerator applications, for example the free electron laser, concern more on the longitudinally sliced property of the beam, such as emittance and energy spread. The beam which contains $1 \times 10^{6}$ macroparticles is dumped at the end of the bypass beam line, and it is analyzed along its longitudinal direction. The sliced normalized emittance and energy spread along the bunch is shown in Fig. 9 (left) and (right). It is demonstrated again that the sliced property of the beam is also preserved. One observes that the slice emittance is $\gamma \epsilon_{x}=\gamma \epsilon_{y}=0.5 \mu \mathrm{m}$ and the slice energy spread is $10 \mathrm{MeV}\left(\sigma_{\delta_{p}}=1 \%\right.$ at a beam energy of $1 \mathrm{GeV}$ ).

The longitudinal phase space is also compared at two different locations in the bypass beam line, say at the injection point and at the end, as shown in Fig. 10.
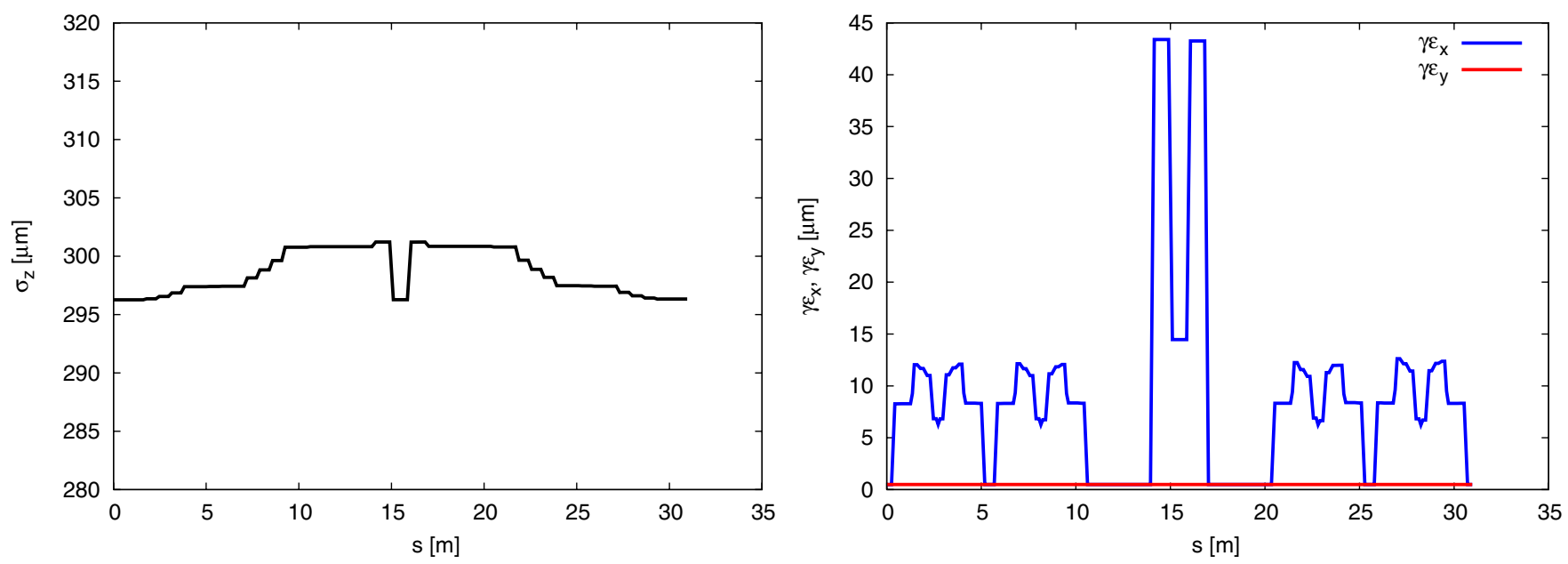

FIG. 8. The rms bunch length and projected normalized emittance evolution along the bypass beam line. Left: The rms bunch length. Right: Projected normalized emittance.
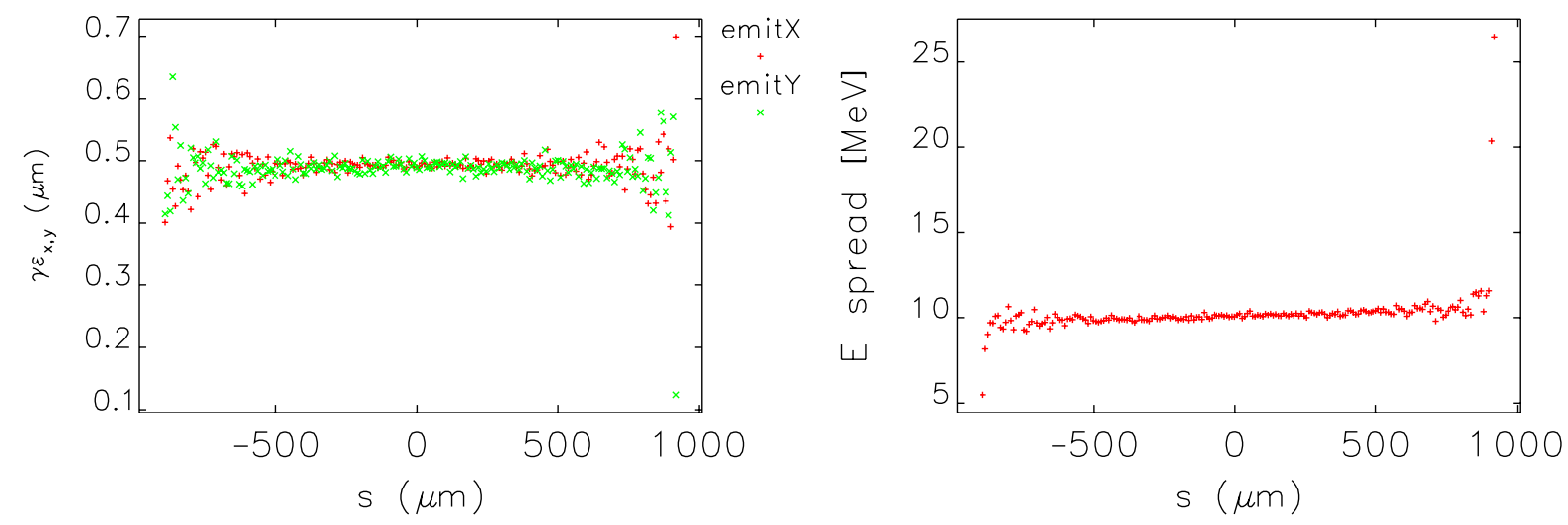

FIG. 9. Sliced normalized emittance and energy spread along the longitudinal direction in a bunch. Left: Sliced normalized emittance. Right: Sliced energy spread. 

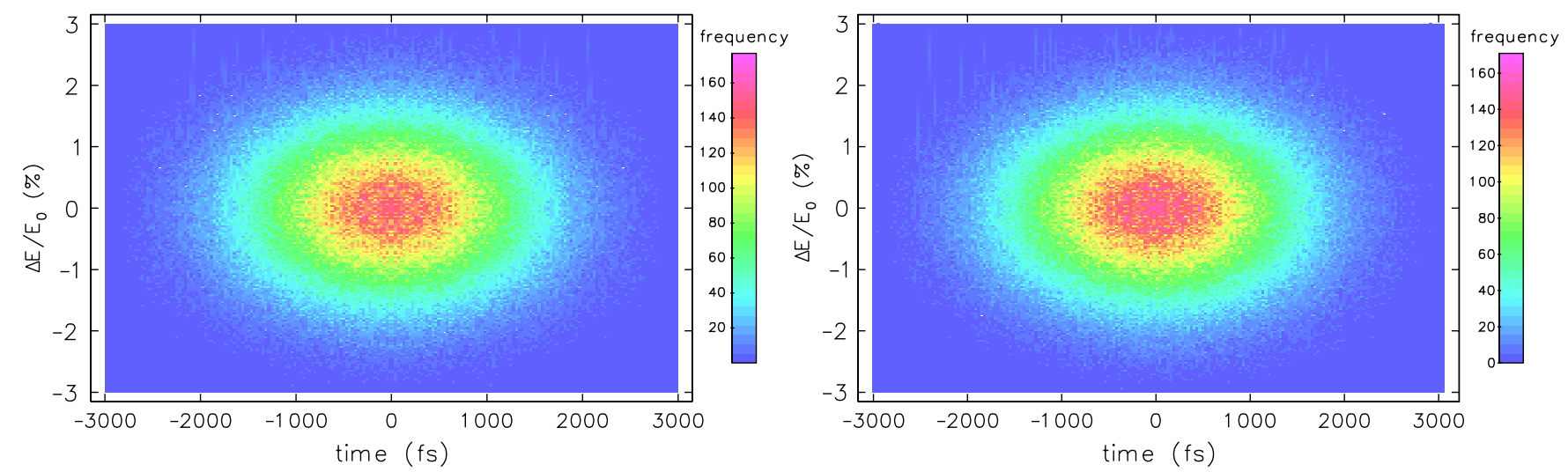

FIG. 10. Longitudinal phase space of the beam. Left: Initial phase space before injected into the bypass. Right: At the end of the bypass beam line.
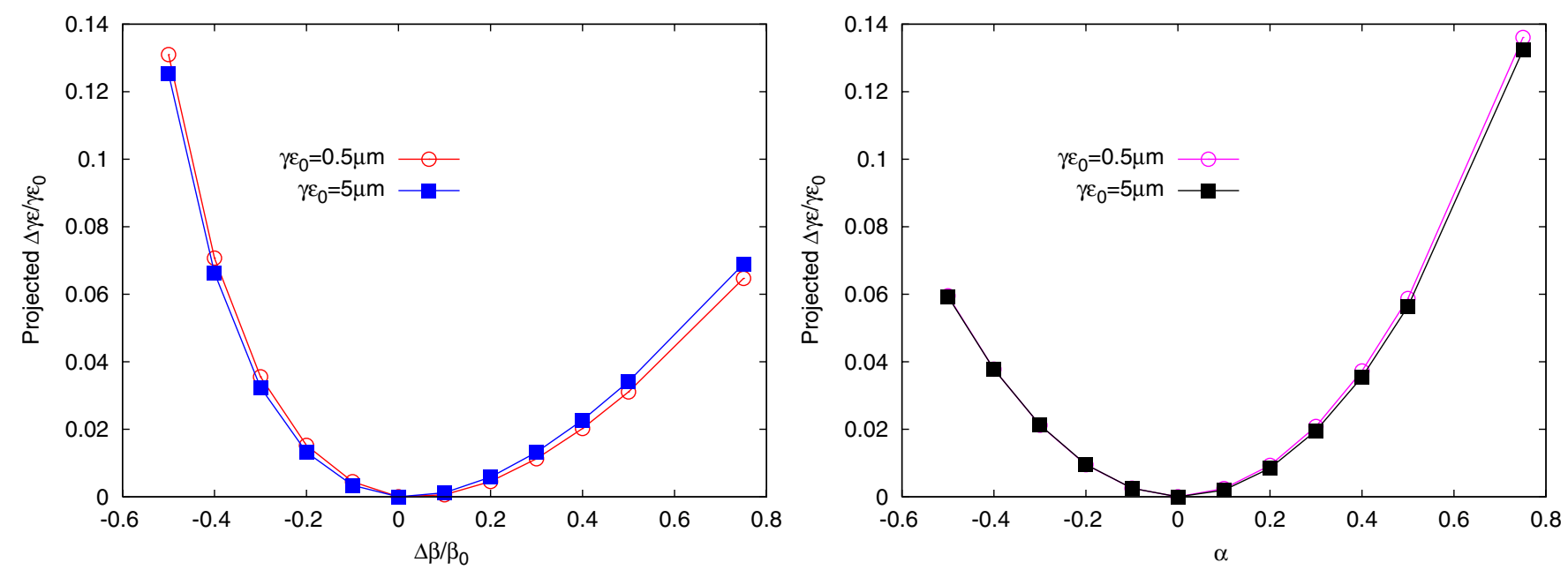

FIG. 11. Left: Relative change in projected normalized emittance versus the mismatched beta function, for two different emittances, $\gamma \epsilon_{x}=\gamma \epsilon_{y}=0.5 \mu \mathrm{m}$ and $\gamma \epsilon_{x}=\gamma \epsilon_{y}=5 \mu \mathrm{m}$. Right: Relative change in projected normalized emittance versus initial alpha function when generating the beam.

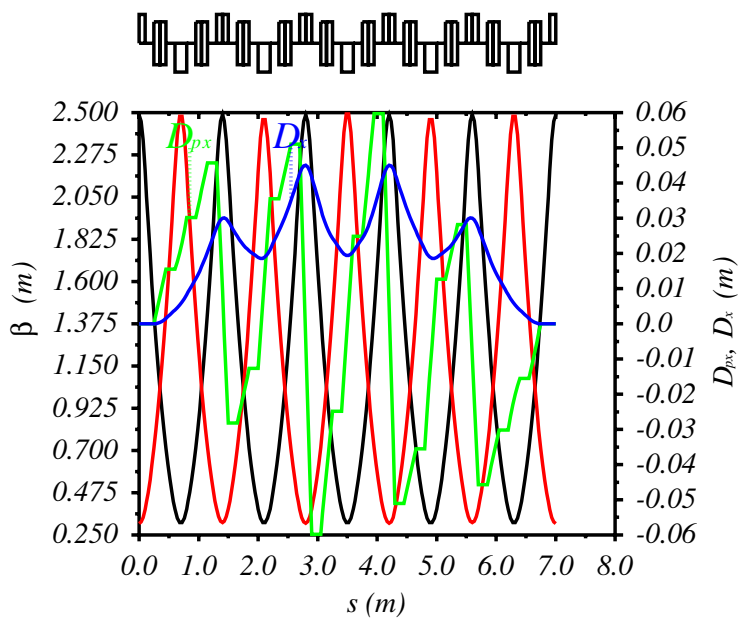

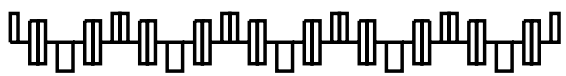

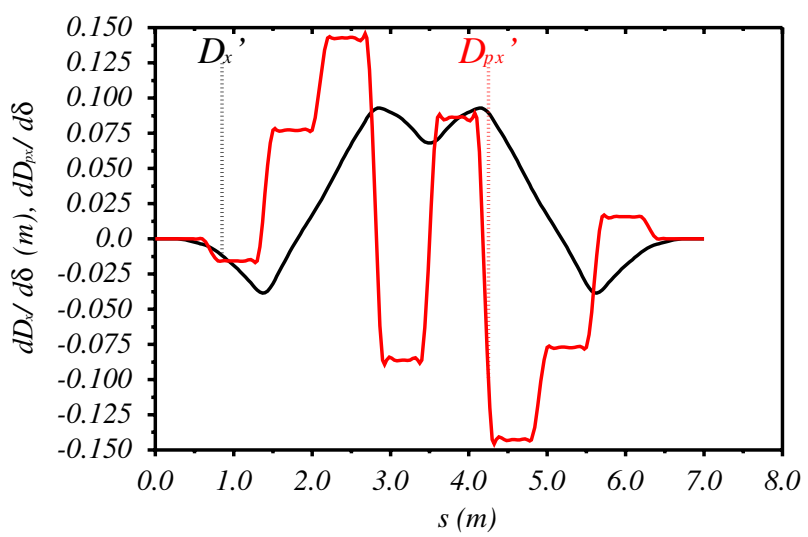

FIG. 12. First order and second-order optics of a supercell composed of five FODO cells. The horizontal and vertical betatron phase advances are both $90^{\circ}$. The four dipole magnets at each end have a bending angle which is roughly a half of the two central dipoles. The dipole strength and phase advance are slightly tuned to generate a second-order achromat. Left: First order dispersion and beta functions. Right: Second-order dispersion $T_{166}$ and angular dispersion $T_{266}$. 
Again, one observes there is a small deformation on the phase space from the large energy spread and a residual second-order dispersion $T_{566}$. The overall effect of the firstorder dispersion $R_{56}$ is zero.

Then an initial beam generated from mismatched Twiss parameters (with respect to the optics) is used and the projected emittance evolution is investigated again under this condition. The matched Twiss parameters are from the periodic solution of the supercell discussed above, which are $\beta_{x 0}=\beta_{y 0}=2 \mathrm{~m}$ and $\alpha_{x 0}=\alpha_{y 0}=0$. An electron beam is generated either by employing a mismatched beta function in the bending plane, or taking a mismatched alpha function in the bending plane. An rms energy spread of $\sigma_{\delta_{p}}=1 \%$ is again used. The tracking simulation is done with two different transverse beam emittance settings, $\gamma \epsilon_{x}=\gamma \epsilon_{y}=0.5 \mu \mathrm{m}$ and $\gamma \epsilon_{x}=\gamma \epsilon_{y}=5 \mu \mathrm{m}$.

The relative variation in projected normalized emittance versus the mismatched beta function is shown in Fig. 11. For the case with a mismatched beta function of $50 \%$, the growth in projected emittance is roughly $10 \%$. Within $\pm 20 \%$, the change in projected emittance is negligible.
One also needs to note that the sliced emittance is preserved much better than the projected emittance. There is no obvious change in slice emittance even with a mismatched beta function of $50 \%$.

\section{CONCLUSION AND DISCUSSION}

A compact supercell is investigated which composes four FODO arc cells with two missing dipoles. A secondorder achromat condition is achieved without sextupoles by tuning the strength of the two dipole magnets at two ends. It is proposed to design dogleg or bypass beam lines with these supercells, which can transport an electron beam with large energy spread and preserve its emittance. The elimination of employing sextupoles makes the tolerance on alignment easier to live with. There are also other possible ways to design such supercells with FODO arc cells which may use more dipole and quadrupole magnets. One possible solution is evolved from the classic dispersion suppressor design approach [8], with the firstorder and second-order optics of its supercell shown in Fig. 12.

\section{ACKNOWLEDGMENTS}

The author would like to thank C. Adolphsen, Y. Cai, A. Chao, P. Emma, J. England, Z. Huang, T. Raubenheimer, W. Wan, M. Woodley, and J. Wu for helpful discussions. This work was supported by the DOE under Contract No. DE-AC02$76 \mathrm{SF} 00515$.

\section{APPENDIX: SECOND-ORDER SUBMATRICES}

The submatrices of the second-order transport matrix are listed below:

$$
\begin{gathered}
\mathbf{T}_{\mathbf{x}, \mathbf{1 1}}=\left(\begin{array}{cccc}
R_{11} & R_{12} & R_{15} & R_{16} \\
R_{21} & R_{22} & R_{25} & R_{26} \\
R_{51} & R_{52} & R_{55} & R_{56} \\
0 & 0 & R_{65} & 1
\end{array}\right) \\
\mathbf{T}_{\mathbf{x}, \mathbf{1 2}=}=\left(\begin{array}{cccccccccc}
T_{111} & T_{112} & T_{115} & T_{116} & T_{122} & T_{125} & T_{126} & T_{155} & T_{156} & T_{166} \\
T_{211} & T_{212} & T_{215} & T_{216} & T_{222} & T_{225} & T_{226} & T_{255} & T_{256} & T_{266} \\
T_{511} & T_{512} & T_{515} & T_{516} & T_{522} & T_{525} & T_{526} & T_{555} & T_{556} & T_{566} \\
0 & 0 & 0 & 0 & 0 & 0 & 0 & 0 & 0 & 0
\end{array}\right) \\
\mathbf{T}_{\mathbf{x}, \mathbf{1 3}}= \\
\left.\begin{array}{ccccccc}
T_{133} & T_{134} & T_{144} \\
T_{233} & T_{234} & T_{244} \\
T_{533} & T_{534} & T_{544} \\
0 & 0 & 0
\end{array}\right) .
\end{gathered}
$$

If one tries to write down every entry in matrix $\mathbf{T}_{\mathbf{2 2},[\mathbf{1 0} \times \mathbf{1 0}]}$, it reads 


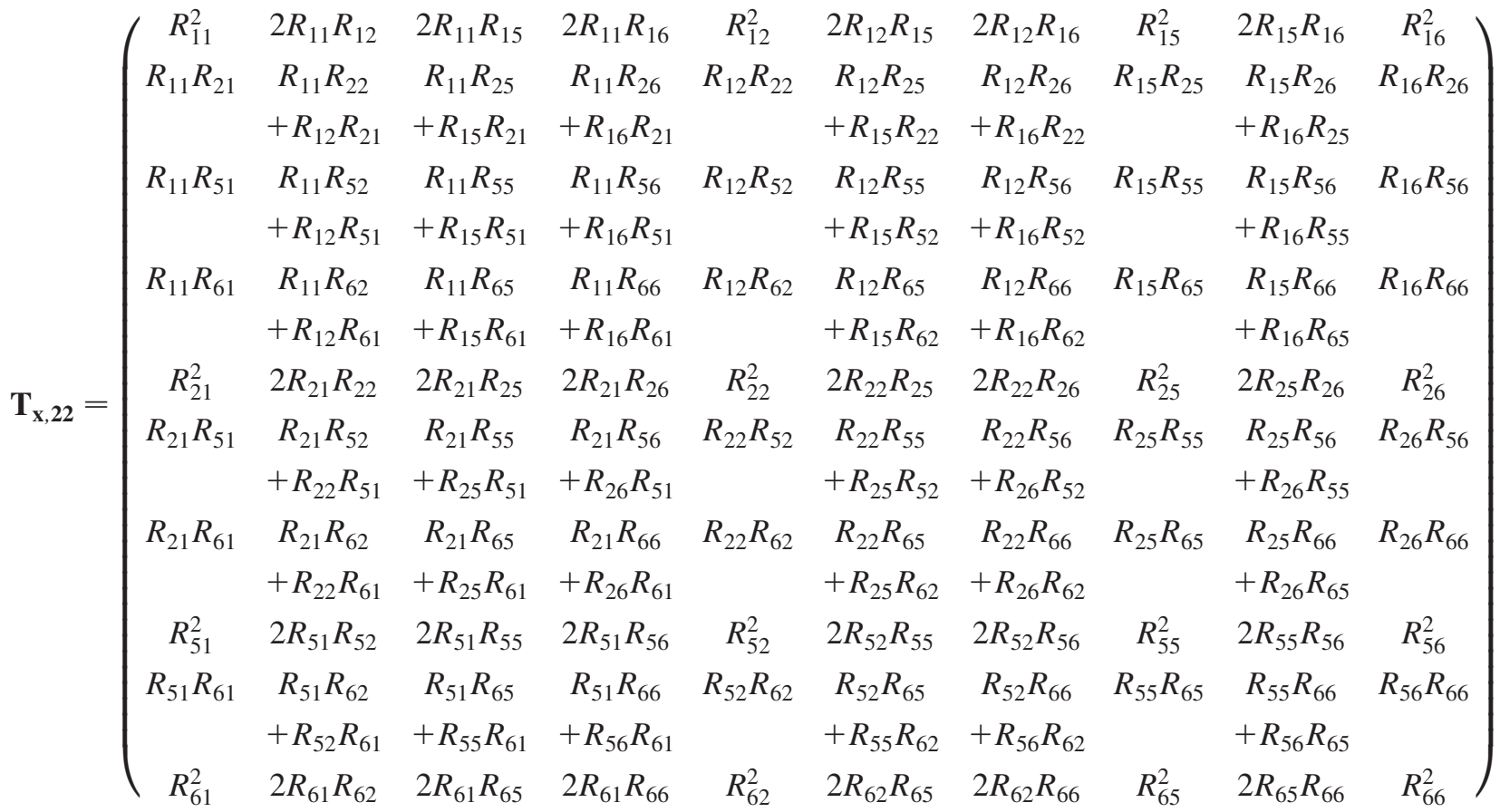

$$
\mathbf{T}_{\mathbf{x}, \mathbf{3 3}}=\left(\begin{array}{ccc}
R_{33}^{2} & 2 R_{33} R_{34} & R_{34}^{2} \\
R_{33} R_{43} & R_{33} R_{44}+R_{34} R_{43} & R_{34} R_{44} \\
R_{43}^{2} & 2 R_{43} R_{44} & R_{44}^{2}
\end{array}\right)
$$

[1] K. Brown, IEEE Trans. Nucl. Sci. 26, 3490 (1979).

[2] W. Wan and M. Berz, Phys. Rev. E 54, 2870 (1996).

[3] M. Borland, ELEGANT: A Flexible SDDS-Compliant Code for Accelerator Simulation (Advanced Photon Source LS287, 2000).

[4] D. Carey, The Optics of Charged Particle Beams (Hardwood Academic, New York, 1987), pp. 21-37, 123-143.
[5] K. Brown, Report No. SLAC-R-075, 1982.

[6] H. Grote and F. C. Iselin, The MAD Program (Methodical Accelerator Design) Version 8.15, Report No. CERN/SL/ 90-13 (AP), 1990.

[7] Maxima, a Computer Algebra System, http://maxima .sourceforge.net/.

[8] A. Chao and M. Tigner, Handbook of Accelerator Physics and Engineering (World Scientific, Singapore, 1998), p. 77. 